\title{
Ultra Low Frequency Wave Produced by Underwater Oscillating Sphere and Its Measurement
}

\author{
Huan Wang ${ }^{1}$, Erzheng Fang ${ }^{1,2,3, *}$, Mingze Wu ${ }^{1}$, Lianjin Hong ${ }^{1,2,3}$ and Zongru $\mathrm{Li}^{1}$ \\ 1 College of Underwater Acoustic Engineering, Harbin Engineering University, Harbin 150001, China; \\ huanw@hrbeu.edu.cn (H.W.); wumingze@hrbeu.edu.cn (M.W.); honglianjin@hrbeu.edu.cn (L.H.); \\ lizongru@hrbeu.edu.cn (Z.L.) \\ 2 Acoustic Science and Technology Laboratory, Harbin Engineering University, Harbin 150001, China \\ 3 Key Laboratory of Marine Information Acquisition and Security (Harbin Engineering University), \\ Ministry of Industry and Information Technology, Harbin 150001, China \\ * Correspondence: fangerzheng@hrbeu.edu.cn
}

Citation: Wang, H.; Fang, E. Wu, M.; Hong, L.; Li, Z. Ultra Low Frequency Wave Produced by Underwater Oscillating Sphere and Its Measurement. J. Mar. Sci. Eng. 2021, 9, 1317. https://doi.org/ 10.3390/jmse9121317

Academic Editor: Giovanni Malara

Received: 21 October 2021

Accepted: 17 November 2021

Published: 23 November 2021

Publisher's Note: MDPI stays neutral with regard to jurisdictional claims in published maps and institutional affiliations.

Copyright: (c) 2021 by the authors. Licensee MDPI, Basel, Switzerland. This article is an open access article distributed under the terms and conditions of the Creative Commons Attribution (CC BY) license (https:// creativecommons.org/licenses/by/ $4.0 /)$.

\begin{abstract}
When an underwater target moves in viscous fluid, it may cause the periodic movement of the surrounding fluid and generate ultra-low-frequency (ULF) gravity waves. The initial domain of the gravitational surface wave propagating above the moving target is named circular wave. This article studies the ULF circular wave generated by underwater oscillating sphere, which will provide basis for underwater long-range target detection. Firstly, the circular wave caused by the sphere oscillation in a finite deep fluid is studied based on the theory of linear potential flow. Meanwhile, the multipole expansion theory is established to solve the circular wave field. Secondly, the interface wave generated by the target oscillation in a two-layer fluid are numerically analyzed by comparison with the free surface fluctuation of a single-layer fluid. The results show that the amplitude of the internal interface displacement (AIID) is smaller than that of the free surface (AFSD). When the sphere is in the lower layer, the layering effect of the fluid has significant influences on the AFSD. Finally, the results of the pool experiment verified that the wave generated by the oscillating sphere is the surface gravity wave. Furthermore, the change trend of the test result is consistent with the simulation result.
\end{abstract}

Keywords: ultra low frequency; sphere oscillation; circular wave; two-layer fluid

\section{Introduction}

With the rapid development of vibration and noise-reduction technology, conventional passive detection based on radiated noises from underwater moving targets is becoming difficult. The frequency band of interest to underwater detection is developing towards very-low-frequency (VLF). The VLF acoustic signal generated by the underwater targets is above $1 \mathrm{~Hz}$ [1,2]. Ambient noises are dominant in this frequency band [3], including marine mammal calls, turbulent pressure fluctuation noises, boating low-frequency noises, impulse noises induced by earthquakes and explosions. Thus, the traditional Gaussian distribution cannot accurately describe this type of noise [4], and it is extremely challenging to detect underwater targets based on the passive detection of acoustic signals. The-VLF underwater acoustic waves interacting with seabed can result inelastic waves, e.g., Scholte wave [5-8]. The Scholte wave propagates along the solid/liquid interface as a cylindrical wave. Since the energy of Scholte wave trapped within a thin layer near the seabed with small attenuations, it can maintain for long-range propagation. Recently, a large number of studies have been carried out based on the joint detection of underwater acoustic and seismic waves [8,9]. In the above detection methods, the regular or irregular movement of the fluid medium is usually not considered, with the influence of medium fluctuations on sound ignored. However, in actual situations, some medium movements have nonnegligible influences on sound propagation. Through the study of these effects, it may 
be helpful for underwater detection. Researchers have observed in the ocean that a large number of natural and artificial environmental acoustic signals are scattered by Ultra Low Frequency (ULF) (mHz) oscillation of moving objects [10]. Further studies have found that the underwater moving target and its ULF field will perturb sound propagation. The ULF field is the gravitational field, which appears to be the surface gravity wave on the free surface. According to marine spectrum standards [11], the ULF wave is defined by the wave frequency between the wind-induced ocean wave and the Rossby wave [12]. It is a hydrodynamic wave with an inertial period (IP) close to the potential field. The most sensitive period is $15 \mathrm{~min}$, and the period ranges from $2 \mathrm{~min}$ to $2 \mathrm{~h}$. Figure 1 [13] shows the physical limits of the ULF range of the marine natural hydrodynamic field indicating that the ULF range is conducive to signal detection and measurement due to low ambient noises in this range.

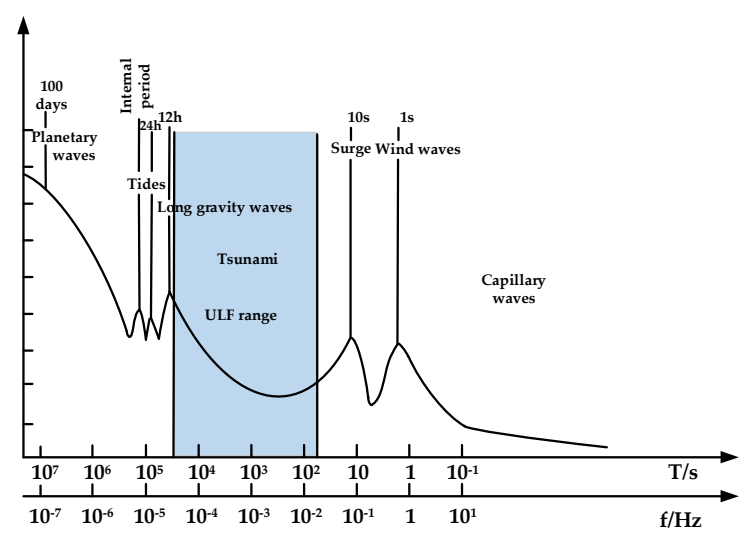

Figure 1. Schematic diagram of the energy spectrum of the marine natural fluid dynamic field.

For the classical long-distance underwater detection at high frequencies, the ULF oscillation of the moving target, scattered sound and extremely low Mach number fluid flow can be ignored. With the development of noise control technology, researchers have focused on the active detection method. The conventional active detection method is complicated and has high power consumption. Experiments have found [14] that the method of the outer field of the aircraft can be used to detect forward scattering. Therefore, ULF waves caused by moving targets have not attracted sufficient attentions in wave scattering theories. For the long-range underwater detection based on the bistatic forward scattering, the ULF waveforms caused by the moving target will modulate acoustical signals $[14,15]$. This discovery provides basis for long-range ULF detection. Figure 2 shows the results in Ref. [15] (p. 101).

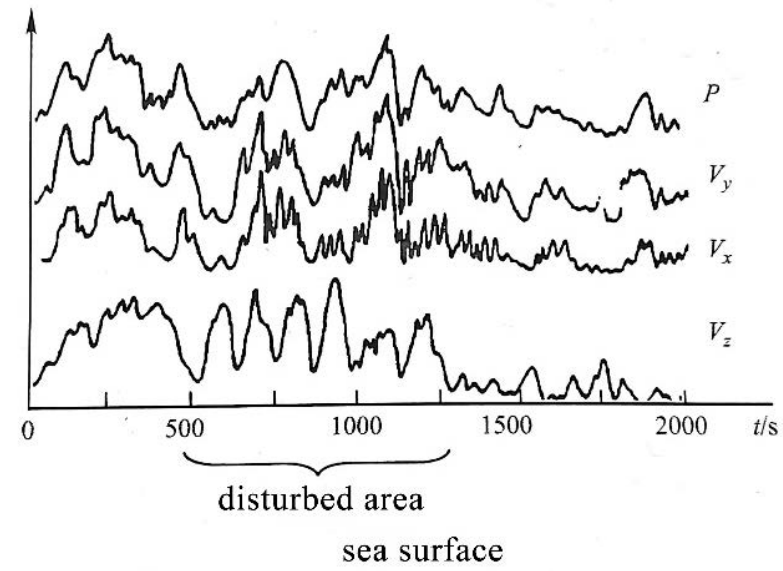

Figure 2. The result of ULF detection [15]. 
In the experiment [15], there are two explanations for the fluctuation of the ULF signal. For the signal between $500 \mathrm{~s}$ to $1250 \mathrm{~s}$, due to the internal waves excited by the wake vortex, the frequency is determined by both the Vaisala frequency and the Coriolis inertial frequency. The signal between $0 \mathrm{~s}$ to $500 \mathrm{~s}$ is related to the low-intensity shock wave formed in front of the target, with the frequency coinciding with that of the vortex formation. The frequency of the three-dimensional vortex formation gives an approximate value $f_{B} \approx u / 4 L$, where $u$ is the target motion speed, and $L$ is the target feature size. Ref. [14] proposes the scattering induced by gravitational surface waves, and defines the frequency of the surface gravity wave as $f_{B} \approx u / 4 L$, where $S_{t}$ is the Strouhal number, $V$ and $u$ are the volume and velocity of the target, Based on Ref. [14], this article gives a third possible explanation. When the target traverses the transmitting and receiving lines, because the surface gravity wave is much faster than the internal wave, it scatters the acoustic signal before the internal wave does. This explains directly the acoustic signal modulation phenomenon in Figure 2. The signal before $500 \mathrm{~s}$ is modulated by surface gravity waves, and that between $500 \mathrm{~s}$ and $1250 \mathrm{~s}$ is modulated by both the surface gravity wave and the internal wave respectively.

When a target moves in a viscous fluid, the uneven flow field excited results in Kelvin wakes, internal waves and surface gravity waves that may be excited in the density discontinuity layer. There has been numerous theoretical studies and numerical simulations for investigating wakes $[16,17]$ and internal waves $[18,19]$ caused by moving targets, but few studies focus on surface gravity waves caused by the oscillations of moving targets [14,20-24]. Yeung [20] used the panel method to study the hydrodynamic problems of floating barges in two-layer fluids with finite depth. Ursell [21] first proposed the multipole expansion method; Thorne [22] derived the hydrodynamic equations of the two-dimensional and three-dimensional target motion; Linton [23] studied the hydrodynamic characteristics of an oscillating sphere in an infinitely deep stratified fluid based on the multipole expansion method; You [24] studied the hydrodynamic characteristics of an oscillating horizontal cylindrical and an oscillating sphere in a two-layer fluid. The results show that the horizontal cylindrical and the sphere oscillation will excite the two modal fluctuations on the free surface and the internal interface. One is the fluctuation of the surface wave mode, and the other is the fluctuation of the internal wave mode. Besides, it shows that within a certain frequency range, the effect of fluid stratification on the hydrodynamic performance of floating structures cannot be ignored. However, the studies mentioned above focus on hydrodynamic characteristics (Added mass and Damping coefficient) in the absence of surface gravity waves. Rahman [25] further studied the velocity field and displacement field generated by a sphere in finite deep water by applying the multipole expansion theory. The initial domain of surface gravitational waves propagating above the moving target is called the circular wave, and the lateral size (radius) of the circular wave is equal to the half-wavelength of the gravitational wave. Semonov [14] analyzed the circular waves caused by target heaves, which are observed in the ocean layer independent of density discontinuities expected from the energy perspective.

This article presents the theoretical study on the circular wave caused by the oscillation of an underwater sphere in a two-layer fluid. Also, a pool test was carried out to verify the theory. Firstly, taking a sphere as model, based on the linear potential flow theory, a multipole expansion theory is established to solve the radiation potential generated by the oscillating motion of a spherical target. Secondly, numerical simulations were carried out to compare the circular wave generated by the heave and sway of the sphere and that of a single layer of fluid. The influence of fluid stratification on the circular wave is discussed. Finally, through the pool test, the vector hydrophone was used to measure the amplitude of the circular wave surface displacement caused by the spheroid oscillation in a finite deep fluid. This study will be significant to the development of the underwater detection. 


\section{Theory}

Due to the D'Alembert's principle and the Karmen vortex street phenomenon, the underwater target will receive not only the resistance in the direction of the water flow, but also the lift force perpendicular to the main flow direction. The force to the target is closely related to the development of the surrounding vortex field, especially the separation and shedding process of the vortex. The lift acting on the target generally has obvious periodic oscillation characteristics. The period of oscillation is twice as that of the Kalman vortex. The lift force causes the target oscillation, the target overcomes the lift force to do work to make the fluid generate ULF surface gravity waves with the same period. During the interaction between the underwater target and the fluid mentioned above, surface gravity wave generated by an underwater moving target is divided into two steps. The first step occurs in the target boundary layer. Here, fluid viscosity cannot be ignored. The fluid interacts with the target to produce the Karman vortex street, and the force causes the target to produce ULF oscillations. The second step is the oscillating motion of the target causing fluid motion, and then generating surface gravity waves. In this process, the fluid viscosity can be ignored. This article mainly studies the second step. The first step is only mentioned, without in-depth discussion and research.

This article studies surface gravity waves and only focus on the circular wave of half wavelength above the target due to the limited wave energy. A two-layer model can be used to simplify the stratified fluid. Different with the case of a uniform fluid, the wave generated by the target oscillation in the two-layer fluid will propagate with two different wave numbers, one mode is the surface wave mode and the other mode is the internal wave mode. This article studies the surface wave modes produced by the oscillating sphere.

\subsection{The Velocity Potential}

The two-layer fluid is assumed to be incompressible and the flow irrotational. Furthermore, the amplitudes of the motions are assumed to be small when compared to the wavelength of the surface gravity wave. The densities of the upper fluid and the lower fluid are respectively $\rho_{1}$ and $\rho_{2}$, the density ratio is $\gamma=\rho_{1} / \rho_{2}$, the depths are $h_{1}$ and $h_{2}$ respectively, the total depth is $h=h_{1}+h_{2}$. A sphere with a radius of $a$ in the upper or lower part of the two layers of fluid, the spherical surface is $S$, the sphere make small amplitude heave and sway motions with circular frequency $\omega$. We establish a rectangular coordinate system and the origin is in the undisturbed internal interface, so the horizontal coordinates are $x$ and $y$ whilst $z$ is vertically upward, as shown in Figure 3 .

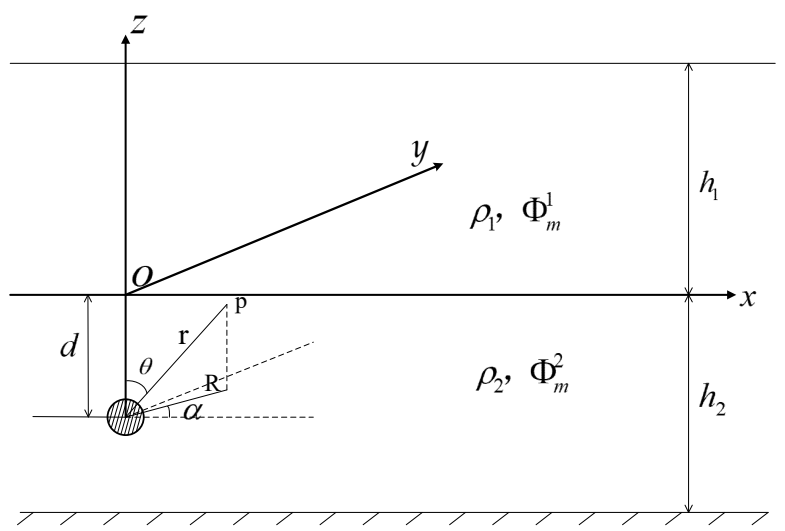

Figure 3. Sphere and coordinate system.

The center of the sphere is at $(0,0, d)$, When the target is in the lower fluid, $d<0$, and when the target is in the upper fluid, $d>0$, the spherical coordinate system is defined as:

$$
x=r \sin \theta \cos \alpha=R \cos \alpha \quad y=r \sin \theta \sin \alpha=R \sin \alpha, \quad z-d=r \cos \theta
$$


The total velocity potential in the upper fluid and the lower fluid are $\Phi^{1}, \Phi^{2}$ respectively, which can be linearly decomposed into:

$$
\Phi^{j}(x, y, z, t)=\operatorname{Re}\left[-i w \sum_{m=0}^{1} \mu_{m} \phi_{m}^{j}(x, y, z) e^{-i w t}\right], j=1,2
$$

- $\mu_{0}$ : the heave amplitude of the sphere.

- $\mu_{1}$ : the sway amplitude of the sphere.

- $\phi_{0}^{j}$ : the spatial parts of the velocity potential at the unit amplitudes of the heave.

- $\phi_{1}^{j}$ : the spatial parts of the velocity potential at the unit amplitudes of the sway.

The velocity potential $\phi_{m}^{j}$ needs to satisfy the Laplace equation in the water domain and the boundary conditions at the water free surface, the internal interface, the water bottom, the sphere surface:

$$
\begin{gathered}
\nabla^{2} \phi_{m}^{j}=0, \quad j=1,2 \quad \text { in the water domain } \\
\frac{\partial \phi_{m}^{1}}{\partial z}=K \phi_{m}^{1}, \quad K=\frac{\omega^{2}}{g} \quad \text { on } z=h_{1} \\
\frac{\partial \phi_{m}^{2}}{\partial z}=0 \text { on } z=-h_{2} \\
\frac{\partial \phi_{m}^{1}}{\partial z}=\frac{\partial \phi_{m}^{2}}{\partial z} \text { on } z=0 \\
\gamma\left(\frac{\partial \phi_{m}^{1}}{\partial z}-K \phi_{m}^{1}\right)=\frac{\partial \phi_{m}^{2}}{\partial z}-K \phi_{m}^{2} \text { on } z=0 \\
\frac{\partial \phi_{m}^{1}}{\partial n}=n_{m} \text { on the sphere surface }
\end{gathered}
$$

- $g$ : the acceleration of gravity.

- $n$ : the unit normal vector of the sphere surface.

- $\quad n_{0}$ : the projection on the $x$ axis.

- $n_{1}$ : the projection on the $z$ axis.

The displacement $\xi^{j}$ of the sphere on the free surface and internal interface when the sphere oscillation is determined by the expressions (9) and (10):

$$
\begin{aligned}
& \Phi_{t}^{1}+g \xi^{1}=0, \quad z=h_{1} \\
& \Phi_{z}^{1}=\Phi_{z}^{2}=\xi^{2}, \quad z=0
\end{aligned}
$$

\subsection{Multipole Potential Expansion}

For the velocity potential that satisfies the preceding conditions, the series solution of the velocity potential can be established according to the method of separating variables. Next, the situation of the sphere in the upper fluid and the lower fluid will be discussed separately.

\subsubsection{Sphere in Lower Fluid Layer}

The multi-pole potential defined in the upper and lower fluids is:

$$
\begin{gathered}
\phi_{n, m}^{1}=\frac{a^{n+1}}{(n-m) !} \int_{0}^{+\infty} k^{n} A(k)\left(v(k) \sinh k\left(z-h_{1}\right)+\cosh k\left(z-h_{1}\right)\right) J_{m}(k R) \mathrm{d} k \\
\phi_{n, m}^{2}=\left(\frac{a}{r}\right)^{n+1} P_{n}^{m}(\cos \theta)+\frac{a^{n+1}}{(n-m) !} \int_{0}^{+\infty} k^{n}\left(B(k) e^{k(z-d)}+C(k) e^{-k(z-d)}\right) J_{m}(k R) \mathrm{d} k
\end{gathered}
$$


where, $v(k)=\omega^{2} / g k, P_{n}^{m}(\cos \theta)=\sin ^{m} \theta \mathrm{d}^{m} P_{n}(\cos \theta) / \mathrm{d}(\cos \theta)^{m}, P_{n}(x)$ is the Legendre polynomial.

The multipole potential (11) in the upper fluid has satisfied the free surface boundary condition (4). In order to make the multipole potential (12) in the lower fluid satisfy the boundary conditions (5)-(7), the following equations need to be used:

$$
\begin{aligned}
& \frac{P_{n}^{m}(\cos \theta)}{r^{n+1}} \cos (m \alpha)=\frac{1}{(n-m) !} \int_{0}^{+\infty} k^{n} e^{-k(z-d)} \cos (m \alpha) J_{m}(k R) \mathrm{d} k, z>d \\
& \frac{P_{n}^{m}(\cos \theta)}{r^{n+1}} \cos (m \alpha)=\frac{(-1)^{m+n}}{(n-m) !} \int_{0}^{+\infty} k^{n} e^{-k(z-d)} \cos (m \alpha) J_{m}(k R) \mathrm{d} k, z<d
\end{aligned}
$$

In order to satisfy the boundary conditions on free surface and internal interface, it could be found that:

$$
\begin{gathered}
A(k)=\frac{-v(k)}{Z(k) \cosh k h_{1} \cosh k h_{2}}\left((-1)^{m+n} e^{-k\left(h_{2}+d\right)}+e^{k\left(h_{2}+d\right)}\right) \\
B(k)=\frac{-Q(k)}{2 Z(k) \cosh k h_{2}}\left((-1)^{m+n} e^{-k h_{2}}+e^{k\left(h_{2}+2 d\right)}\right) \\
C(k)=\frac{-1}{2 Z(k) \cosh k h_{2}}\left((-1)^{m+n} e^{-k\left(h_{2}+2 d\right)} V(k)+Q(k) e^{-k h_{2}}\right)
\end{gathered}
$$

where

$$
\begin{gathered}
Z(k)=\left(1+\gamma t_{1} t_{2}\right)[v(k)]^{2}-\left(t_{1}+t_{2}\right) v(k)+(1-\gamma) t_{1} t_{2} \\
Q(k)=\left(1-\gamma t_{1}\right)[v(k)]^{2}+\left(1-t_{1}\right) v(k)-(1-\gamma) t_{1} \\
V(k)=-\left(1+\gamma t_{1}\right)[v(k)]^{2}+\left(1+t_{1}\right) v(k)-(1-\gamma) t_{1}
\end{gathered}
$$

where, $t_{1}=\tanh k h_{1}, t_{2}=\tanh k h_{2}$, in the formulas (11) and (12), the integral represents the path integral under the two poles $k=k_{1}$ and $k=k_{2}, k_{1}, k_{2}$ is the root of the equation that satisfies the following dispersion relation:

$$
v(k)=\frac{t_{1}+t_{2}+(-1)^{n+1} \sqrt{\left(t_{1}+t_{2}\right)^{2}-4(1-\gamma) t_{1} t_{2}\left(1+\gamma t_{1} t_{2}\right)}}{2\left(1+\gamma t_{1} t_{2}\right)}, n=1,2
$$

When the depth of the upper fluid is 0 , the two fluids degenerate into a single fluid, and the dispersion Equation (20) is simplified to:

$$
\omega^{2}=\operatorname{gktanh}(k h)
$$

Use the following identity (see Thorne 1953):

$$
e^{ \pm k(z-d)} J_{m}(k R)=( \pm 1)^{m} \sum_{s=m}^{+\infty} \frac{( \pm k R)}{(s+m) !} P_{s}^{m}(\cos \theta)
$$

The velocity potential (12) can be expressed as:

$$
\phi_{n, m}^{2}=\left(\frac{a}{r}\right)^{n+1} P_{n}^{m}(\cos \theta)+\sum_{s=m}^{+\infty} A_{n s}^{m}\left(\frac{r}{a}\right)^{s} P_{s}^{m}(\cos \theta)
$$

where the auxiliary function is expressed as:

$$
A_{n s}^{m}=\frac{a}{(n-m) !(s+m) !}\left(\begin{array}{c}
\int_{0}^{+\infty}(a k)^{n+s}\left(B(k)+(-1)^{m+s} C(k)\right) \mathrm{d} k \\
+i \pi \sum_{i=1}^{2}\left(a k_{i}\right)^{n+s}\left(B^{0}\left(k_{i}\right)+(-1)^{m+s} C^{0}\left(k_{i}\right)\right)
\end{array}\right)
$$


- " 0 ": the residue of the corresponding function at the pole.

\subsubsection{Sphere in Upper Fluid Layer}

The multipole potential defined in the upper and lower fluids is:

$$
\begin{gathered}
\phi_{n, m}^{1}=\left(\frac{a}{r}\right)^{n+1} P_{n}^{m}(\cos \theta)+\frac{a^{n+1}}{(n-m) !} \int_{0}^{+\infty} k^{n}\left(B(k) e^{k(z-d)}+C(k) e^{-k(z-d)}\right) J_{m}(k R) \mathrm{d} k \\
\phi_{n, m}^{2}=\frac{a^{n+1}}{(n-m) !} \int_{0}^{+\infty} k^{n} A(k) \cosh k\left(z+h_{2}\right) J_{m}(k R) \mathrm{d} k
\end{gathered}
$$

Equation (24) satisfies the free surface boundary condition (4). From the bottom boundary condition (5), the internal interface conditions (6) and (7) and the relationship (13), corresponding coefficients could be expressed as:

$$
\begin{gathered}
A(k)=\frac{-\gamma v(k)}{z(k) \cosh k h_{1} \cosh k h_{2}}\left((1+v(k)) e^{-k\left(h_{1}-d\right)}+(-1)^{n+m}(1-v(k)) e^{k\left(h_{1}-d\right)}\right) \\
B(k)=\frac{1+v(k)}{2 z(k) \cosh k h_{1}}\left(V(k) e^{k\left(2 d-h_{1}\right)}+(-1)^{m+n} Q(k) e^{-k h_{1}}\right) \\
C(k)=\frac{Q(k)}{2 z(k) \cosh k h_{1}}\left((1+V(k)) e^{-k h_{1}}+(-1)^{m+n}(1-v(k)) e^{-k\left(2 d-h_{1}\right)}\right)
\end{gathered}
$$

where

$$
\begin{aligned}
& Q(k)=t_{2}-v(k)-\gamma t_{2}(1-v(k)) \\
& V(k)=t_{2}-v(k)-\gamma t_{2}(1+v(k))
\end{aligned}
$$

Using formula (22), (24) can be rewritten as:

$$
\phi_{n, m}^{1}=\left(\frac{a}{r}\right)^{n+1} P_{n}^{m}(\cos \theta)+\sum_{s=m}^{+\infty} A_{n s}^{m}\left(\frac{r}{a}\right)^{s} P_{s}^{m}(\cos \theta)
$$

\subsection{Circular Wave}

According to the Ref. [24], in two-layer fluid, whether it is the heave or sway motion of a cylinder, two modal fluctuations will be excited on the free surface and the inner interface: one is corresponding to the wave number as $k_{1}$, the other is the fluctuation of the internal wave mode corresponding to the wavenumber $k_{2}$. However, there are only the surface wave mode of the wavenumber $k_{1}$ in the single-layer fluid. The paper mainly studies the surface wave with wavenumber $k_{1}$. The multipole potential is the solution of the governing equation. The equation is singular at the center of the sphere. It satisfies all the boundary conditions of the problem except on the surface of the sphere. And at the horizontal distance from the singular point, the multipolar potential is in the form of a cylindrical wave and the internal interface and free surface are in the form of a circular wave. The multipole potential constructed above is used in combination with the boundary conditions of the sphere surface to calculate the circular wave generated by the sphere oscillation.

The velocity generated by the heave and sway motion of the sphere can be expressed as $\mathbf{U}_{0}=\operatorname{Re}\left\{U e^{i w t}\right\} \mathbf{k}$ and $\mathbf{U}_{1}=\operatorname{Re}\left\{U e^{i w t}\right\} \mathbf{i}$, where $\mathbf{k}$ is the unit vector pointing to the $z$-axis, $\mathbf{i}$ is the unit vector pointing to the $x$-axis, and the potential is expressed as $\phi_{0}$ and $\phi_{1}$, From Legendre function $P_{1}^{0}(\cos \theta)=\cos \theta, P_{1}^{1}(\cos \theta)=\sin \theta$, the boundary conditions (8) for the spheroid heave and sway can be expressed as:

$$
\frac{\partial \phi_{m}}{\partial r}=U P_{1}^{m}(\cos \theta) \cos m \alpha, \quad r=a
$$


According to the multipole potential constructed above, the radiation potential of the upper and lower fluids can be expressed as:

$$
\phi_{m}^{j}=a \cos m \alpha \sum_{n=1}^{\infty} b_{n, m} \phi_{n, m}^{j}, m=0,1, j=1,2
$$

From the boundary conditions (31) and the orthogonality of Legendre function, the relationship of unknown coefficients is obtained:

$$
b_{s, m}-\frac{s}{s+1} \sum_{n=1}^{+\infty} A_{n s}^{m} b_{n, m}=-\frac{\delta_{1 s}}{2} \quad s \geq 1, \quad m=0,1,2 \ldots
$$

- $\delta_{n s}$ : the Kronecker delta.

The above-mentioned multipole expansion is only effective in the range of $r<2 *|f|$ (Thorne, 1953). It can be seen from (32) that in the upper and lower fluids, the far-field interface wave radiation potential can be expressed

$$
\phi_{m}^{j} \sim Z^{j}\left(k_{1}, z\right) E\left(k_{1}\right) H_{m}^{1}\left(k_{1} r\right), m=0,1, j=1,2
$$

where:

$$
\begin{gathered}
Z^{1}\left(k_{1}, z\right)=\frac{v\left(k_{1}\right) \sinh k_{1}\left(z-h_{1}\right)+\cosh k_{1}\left(z-h_{1}\right)}{\cosh k_{1} h_{1}\left(1-\tanh k_{1} h_{1} / v\left(k_{1}\right)\right)} \\
Z^{2}\left(k_{1}, z\right)=\frac{v\left(k_{1}\right)}{\sinh k_{1} h_{2}} \cosh k_{1}\left(z+h_{2}\right)
\end{gathered}
$$

When the target is in the lower fluid,

$$
E_{m}\left(k_{1}\right)=a \cos m \alpha \frac{\pi i\left(v\left(k_{1}\right)-\tanh k_{1} h_{1}\right)}{Z^{\prime}\left(k_{1}\right) \cosh k_{1} h_{2}} \sum_{n=1}^{\infty} \frac{a^{n+1}}{(n-m) !} k_{1}^{n} b_{n, m}\left((-1)^{m+n} e^{-k_{1}\left(h_{2}+d\right)}+e^{k_{1}\left(h_{2}+d\right)}\right)
$$

When the sphere is in the upper fluid,

$$
E_{m}\left(k_{1}\right)=-a \cos m \alpha \frac{\pi i \gamma \tanh k_{1} h_{2}}{Z^{\prime}\left(k_{1}\right) \cosh k_{1} h_{1}} \sum_{n=1}^{\infty} \frac{a^{n+1}}{(n-m) !} k_{1}^{n} b_{n, m}\left(\left(1+v\left(k_{1}\right)\right) e^{-k\left(h_{1}-d\right)}+(-1)^{n+m}\left(1-v\left(k_{1}\right)\right) e^{k\left(h_{1}-d\right)}\right)
$$

where, $Z^{\prime}\left(k_{1}\right)$ is the derivative of $Z\left(k_{1}\right)$ over $k_{1}$.

It can be obtained from the formula (34) that no matter the target heaves or sways, it excites the radiation potential of the surface wave mode of wavenumber $k_{1}$. Next, this article studies the circular wave, which derives from the sphere heaving and swaying on the free surface and the internal interface. According to Formulas (2), (9) and (10), the amplitude of vertical displacements excited on the free surface and internal interface of the $m$-th oscillating mode of the sphere are:

$$
\begin{array}{ll}
\xi^{1}=\operatorname{Re}\left[\sum_{m=0}^{1} \mu_{m} \xi_{m}^{1} e^{-i w t}\right], & \xi_{m}^{1}=\left.\frac{\partial \phi_{m}^{1}}{\partial z}\right|_{z=h_{1}} \\
\xi^{2}=\operatorname{Re}\left[\sum_{m=0}^{1} \mu_{m} \xi_{m}^{2} e^{-i w t}\right], & \xi_{m}^{2}=\left.\frac{\partial \phi_{m}^{2}}{\partial z}\right|_{z=0}
\end{array}
$$

- $\xi_{0}^{j}, \xi_{1}^{j}$ : the AFSD and AIID excited under the unit amplitude of the sphere heave and sway respectively.

Define the value of AFSD and AIID: $A F S D=\xi_{0}^{j}, A I I D=\xi_{1}^{j}$.

It can be obtained from (34) that the far-field form of AFSD and AIID.

$$
\xi_{0}^{1} \sim \pm c_{0}^{1} e^{i k_{1} R}, \quad \xi_{1}^{1} \sim \pm c_{1}^{1} e^{i k_{1} R}
$$




$$
\xi_{0}^{2} \sim \pm c_{0}^{2} e^{i k_{1} R}, \quad \xi_{1}^{2} \sim \pm c_{1}^{2} e^{i k_{1} R}
$$

where

$$
c_{m}^{1}=\frac{K E_{m}\left(k_{1}\right)}{\cosh k_{1} h_{1}\left(1-\tanh k_{1} h_{1} / v\left(k_{1}\right)\right)}, \quad c_{m}^{2}=K E_{m}\left(k_{1}\right)
$$

\section{Simulation and Analysis}

For the infinite dimension of a linear relationship, it can be solved by the truncation method. In numerical calculations, the relational expression is truncated at $n=N$ and $N \times N$ equations are used to calculate the coefficients $b_{n, m}$. Studies have verified that the computation error are convergent when $N=4$. Thus, in the following numerical calculations, $N=4$ be regarded as the criterion.

Curves of AID for spheres in the lower fluid layer in both heave and sway are shown in Figures 4 and 5, and compared with the AFSD produced by a single layer of fluid. In all the curves, when the sphere is in the upper fluid, $\gamma=0.97, t=0, R=0, h_{1} / a=h_{2} / a=5$, $d / a=3,3.5$, corresponding to $d / a=-1.5,-2$ of a single layer of fluid. when the sphere is in the lower fluid, $d / a=-2,-3$, corresponding to $d / a=-7,-8$ of a single layer of fluid. $S$ represents the AFSD of the single-layer fluid, S1 and S2 represent the AFSD and AIID of the two-layer fluid, respectively.

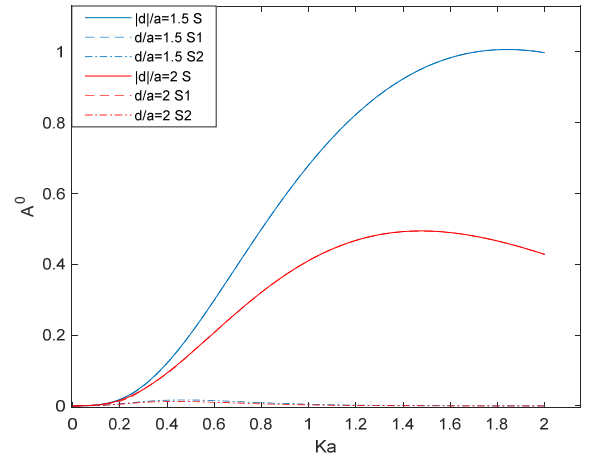

(a)

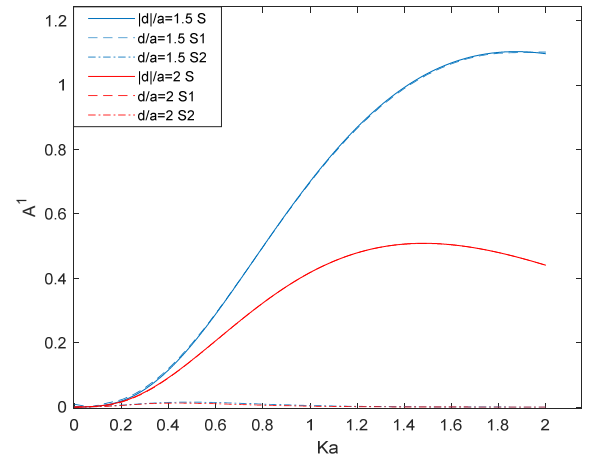

(b)

Figure 4. Sphere is in the upper fluid, the AID caused by the movement of the sphere $A^{0}$ (heave) and $A^{1}$ (sway) change with Ka. (a) $m=0$, (b) $m=1$.

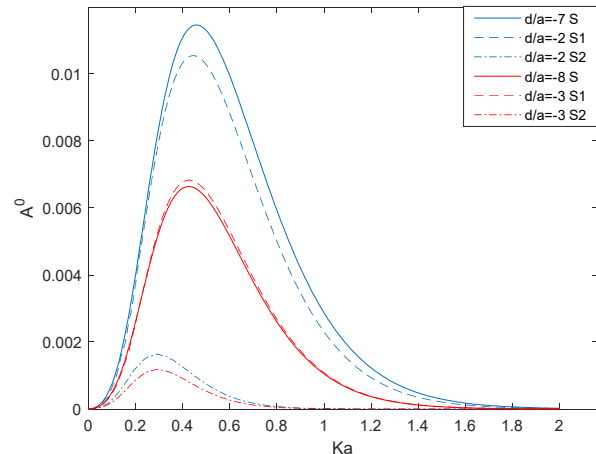

(a)

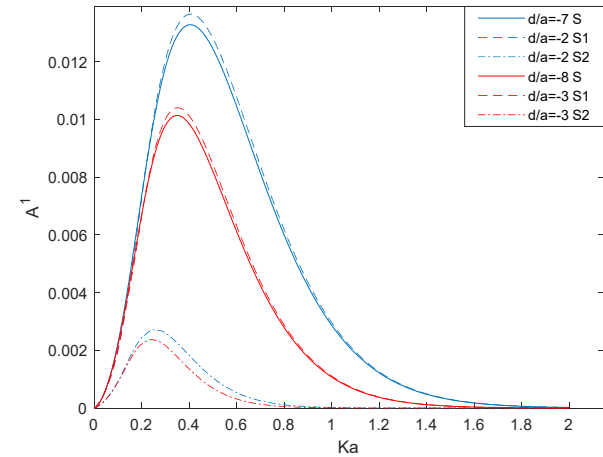

(b)

Figure 5. Sphere is in the lower fluid, the AID caused by the movement of the sphere $A^{0}$ (heave) and $A^{1}$ (sway) change with Ka. (a) $m=0$, (b) $m=1$.

It can be obtained from Figures 4 and 5 that the AID of the surface wave mode with a wavenumber of $k 1$ caused by the spherical heave and sway motion first increases and then decreases as the frequency $K a$ increases, for a given target depth. Also, large target depths are associated with small AID. The AIID is smaller than AFSD. When the sphere is in the upper fluid, the layering effect of the fluid has negligible impacts on the AFSD 
caused by the sphere sway and heave motion. On the contrary, when the sphere is in the lower fluid, the layering effect of the fluid has significant impacts on the ASFD. Therefore, it is important to consider the stratification effect of fluid.

Figures 6 and 7 plots the AID at different internal interface positions. When the sphere is in the upper fluid, the distance between the sphere and the bottom is $\left(h_{2}+d\right) / a=8$, $h_{1} / a=4,5,6$, when the sphere is in the lower fluid, $\left(h_{2}+d\right) / a=3, h_{1} / a=4,5,6$. S1 and S2 represent the ASFD and AIID of the two-layer fluid.

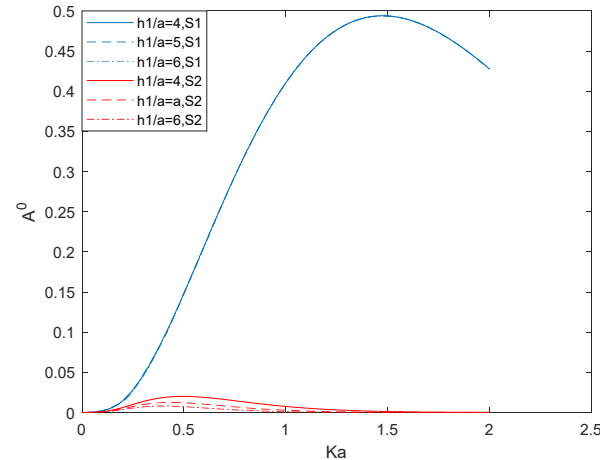

(a)

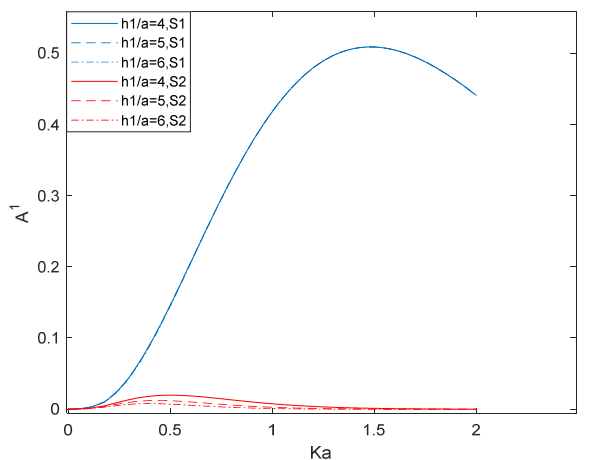

(b)

Figure 6. Sphere is in the upper fluid, the AID caused by the movement of the sphere $A^{0}$ (heave) and $A^{1}$ (sway) change with Ka. (a) $m=0$, (b) $m=1$.

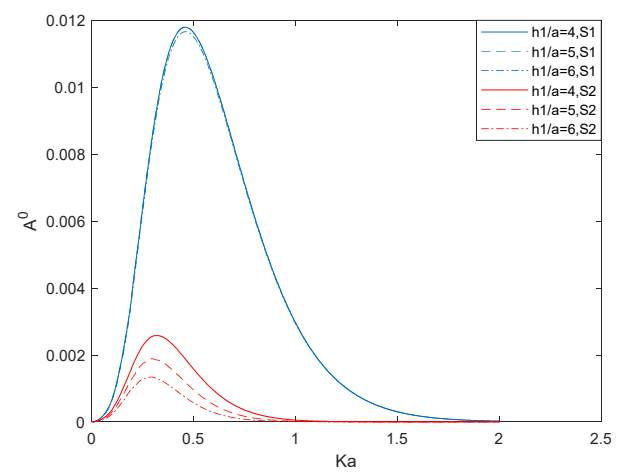

(a)

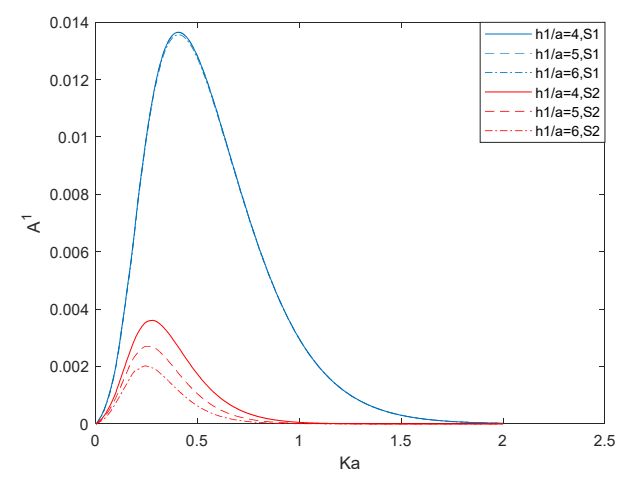

(b)

Figure 7. Sphere is in the lower fluid, the AID caused by the movement of the sphere $A^{0}$ (heave) and $A^{1}$ (sway) change with $K a$. (a) $m=0$, (b) $m=1$.

The results show that the position of the internal interface barely affects the ASFD caused by the sphere sway and heave motion. On the contrary, the position of the internal interface has a great influence on the AIID; with the increase of the depth of the internal interface, the AIID decreases.

Next, the circular wave generated at the free surface when the sphere is in heave and sway motion is examined. Set the depth of the liquid and the position of the sphere to be $h / a=50$ and, respectively.

Figure 8 demonstrates the apparent symmetric circular wave generated by the heave motion in comparison with the anti-symmetric pattern observed in the sway motion scenario. Figure 9 shows the AFSD caused by the movement of the sphere $A^{0}$ (heave) and $A^{1}$ (sway) change with $f$. Result shows that in the case of a given target radius, AFSD increases first and then decreases as the frequency increases, for a given target depth. Also, large target depths are associated with small AID. 


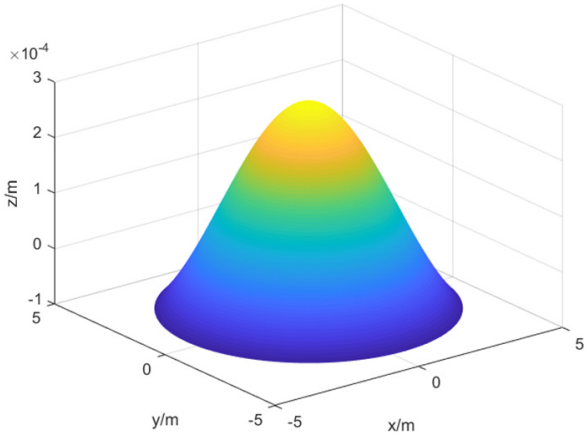

(a)

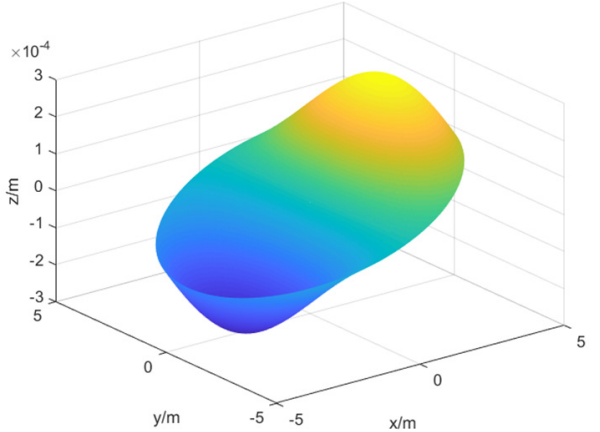

(b)

Figure 8. Free surface circular waves caused by sphere heaving and swaying. (a) $m=0,(\mathbf{b}) m=1$.

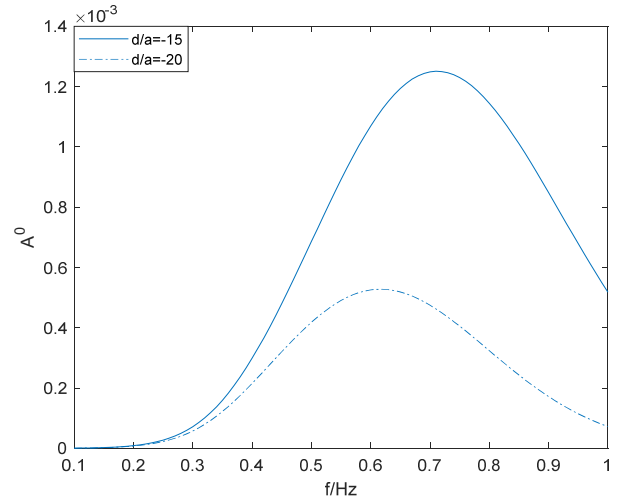

(a)

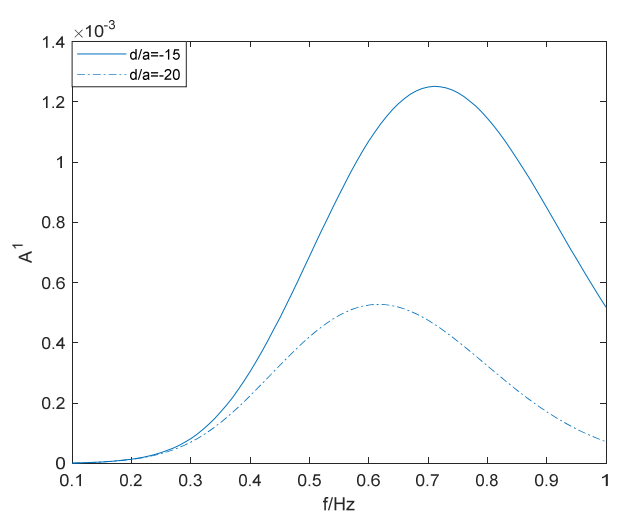

(b)

Figure 9. The AFSD caused by the movement of the sphere $A^{0}$ (heave) and $A^{1}$ (sway) change with $f$. (a) $m=0,(\mathbf{b}) m=1$.

\section{Experimental Verification}

\subsection{Test System}

In order to validate the above-mentioned multipole expansion theory to calculate the circular wave, an experiment was carried out in a pool with a sizes of $10 \mathrm{~m} \times 5 \mathrm{~m} \times 5 \mathrm{~m}$. A rigid sphere with a radius of $0.1 \mathrm{~m}$ was tested and its oscillation frequency was about $0.43 \mathrm{~Hz}, 0.5 \mathrm{~Hz}$, and $0.6 \mathrm{~Hz}$. A surface gravity wave of with a wave velocity of $\sqrt{\mathrm{gh}}=7 \mathrm{~m} / \mathrm{s}$ was generated. Due to the limitation of the test conditions, although the test frequency is in the range of $0.1-1 \mathrm{~Hz}$, the principle of the surface gravity wave generated by the sphere oscillation in this frequency band is consistent with that of ULF range, which can also prove the multipole potential theory. The test system consists of three parts, namely the motion source part, the sensor part and the acquisition part. The sphere was suspended from the walking mechanism for heaving and swaying motions under water. For the sensor part, since the sound pressure channel of a vector hydrophone was used to collect data by sensing variations in pressures, it can collect both acoustic and hydrodynamic signals which can be separated simply in the frequency domain. The sensor part consists of a vector hydrophone with a sensitivity of $-220 \mathrm{~dB} @ 1 \mathrm{~m}$ ref $1 \mu \mathrm{P}$ in the range of $0.1-1 \mathrm{~Hz}$, a B\&K8103 hydrophone, a B\&K2651 amplifier, The acquisition part is Pulse collector (sampling rate $65,535 \mathrm{~Hz}$ ) and laptop. The test system is shown in Figure 10. 


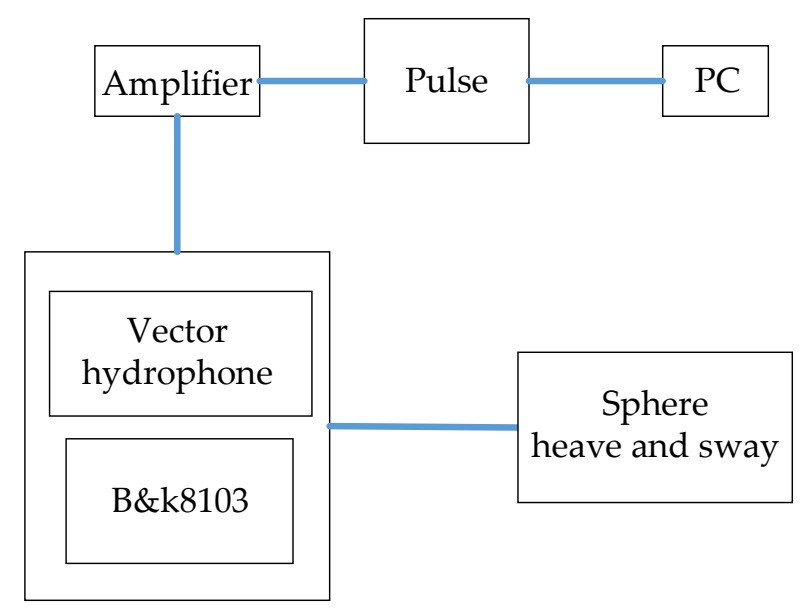

Figure 10. Block diagram of test system.

\subsection{Experimental Analysis}

The test layout is shown in Figure 11. The sphere was at a depth of $z$, with the hydrophone placed at a certain distance from it to receive the signal. The distance between the 8103 and the vector hydrophone is $d d$.

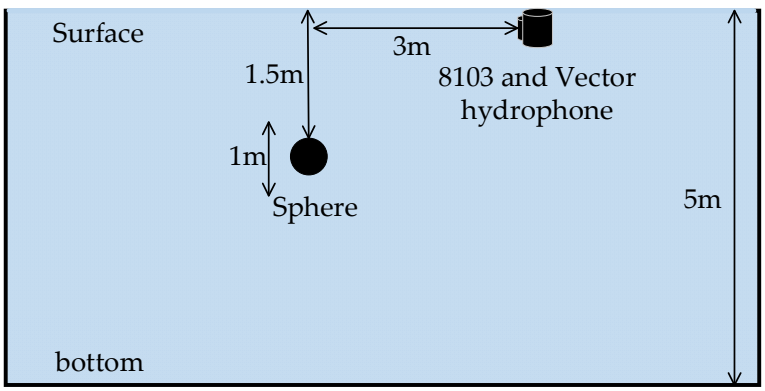

(a)

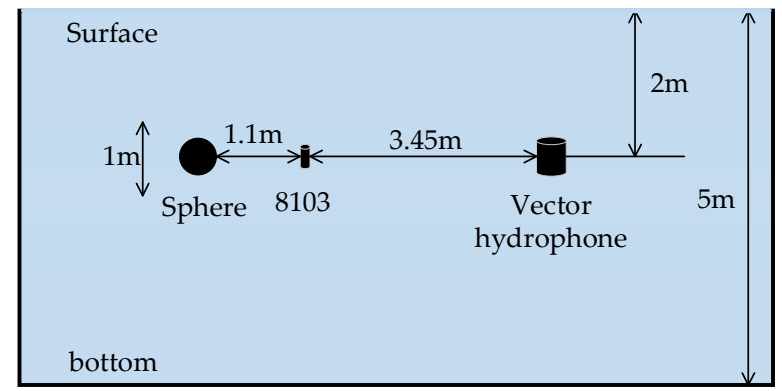

(b)

Figure 11. Schematic diagram of deployment. (a) $d d=0 \mathrm{~m},(\mathbf{b}) d d=3.45 \mathrm{~m}$.

Since the frequency of the gravity wave is much smaller than the sampling rate, it is necessary to downsample the signal to save the data storage space and processing time. Preprocess of the signals collected by the vector hydrophone and 8103 includes data selection, downsampling, and low-pass filtering. For the system deployed according to Figure 11a, the time domain pressure signals received by the vector hydrophone and 8103 are shown in Figures 12a and 13a. After down sampling, Figures 12b and 13b are obtained, and then low-pass filtering is performed to obtain Figures 12c and 13c. The pressure signals in Figures 14 and 15 are collected by the system deployed according to Figure $11 \mathrm{~b}$ and obtained through the above processing methods. The slow change in Figure 15 is a linear or slowly changing trend error generated in the time series due to some reasons in the test system, but it has no effect on the results of this article. 

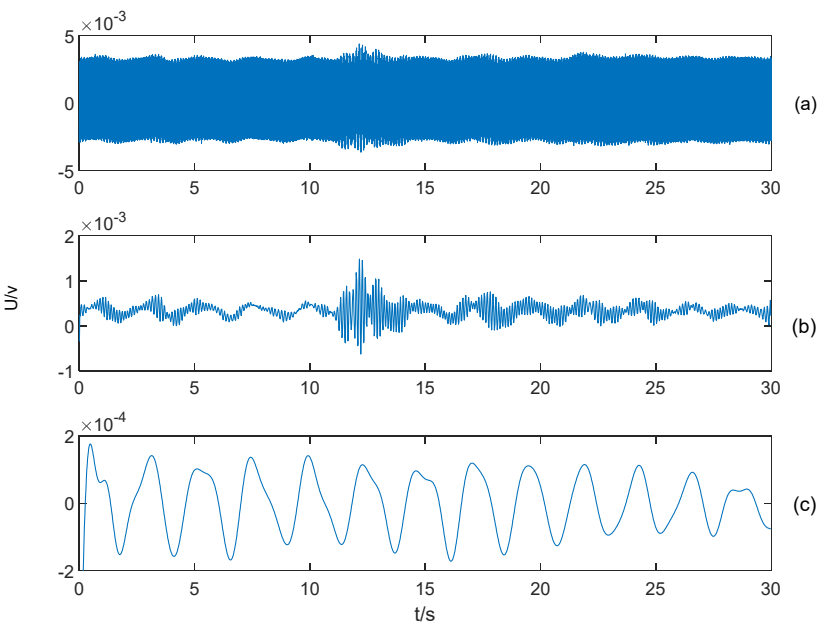

Figure 12. Vector hydrophone P channel time domain waveform. (a) original signal, (b) downsampled signal, (c) low-pass filtered signal.
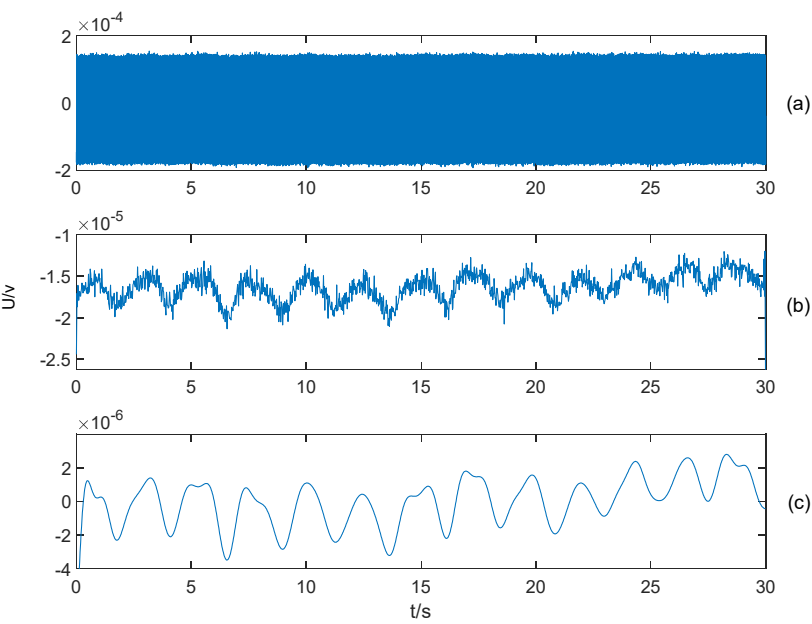

Figure 13. 8103 time domain waveform. (a) original signal, (b) downsampled signal, (c) low-pass filtered signal.

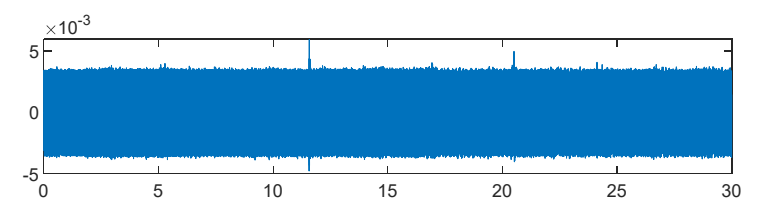

(a)
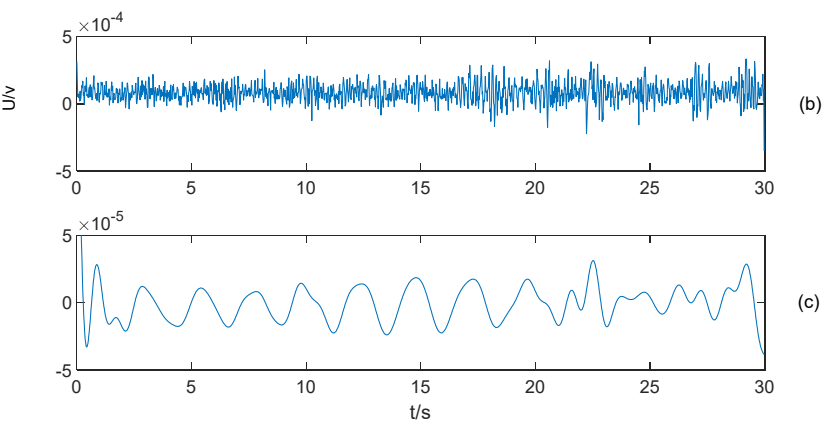

Figure 14. Vector hydrophone P channel time domain waveform. (a) original signal, (b) downsampled signal, (c) low-pass filtered signal. 

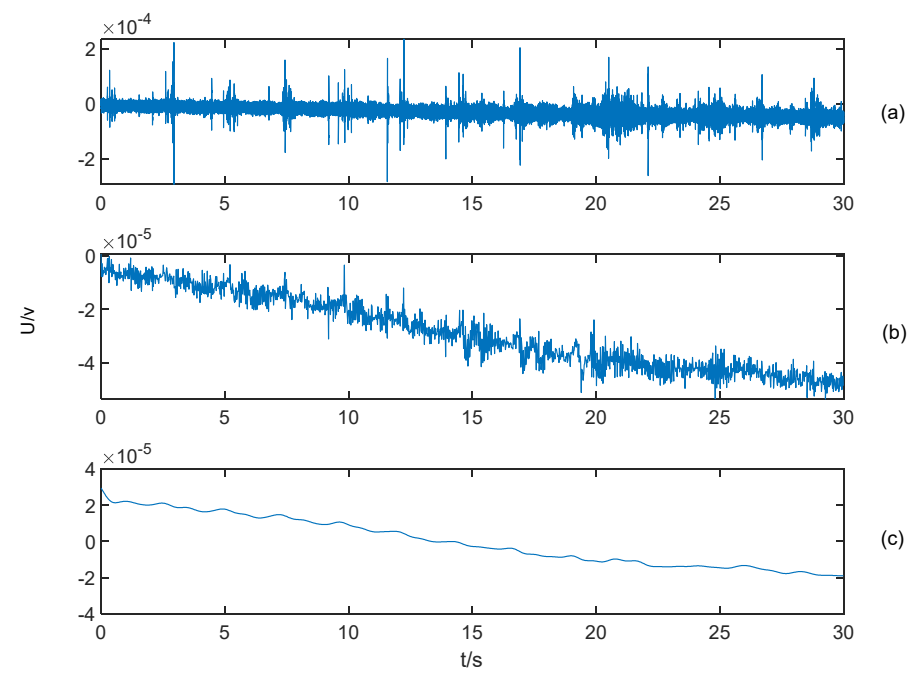

Figure 15. 8103 time domain waveform. (a) original signal, (b) downsampled signal, (c) low-pass filtered signal.

Through the cross-correlation processing of the vector hydrophone and the 8103 pressure signal, the correlation coefficients obtained from Figures $12 \mathrm{c}$ and $13 \mathrm{c}$ are shown in Figure 16a. The correlation coefficients obtained from Figures 14c and 15c are shown in Figure 16b.

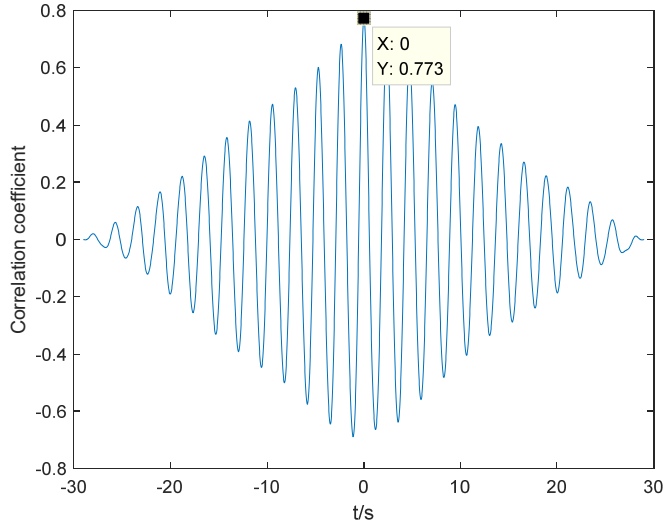

(a)

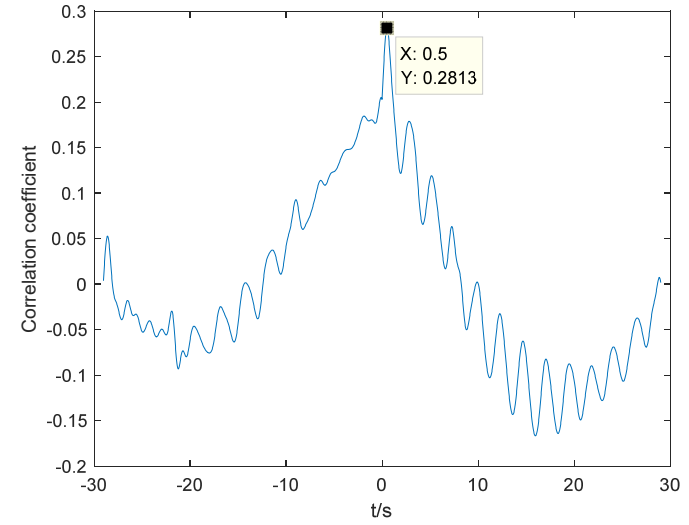

(b)

Figure 16. Time-domain signal correlation coefficient. (a) $d d=0 \mathrm{~m},(\mathbf{b}) d d=3.45 \mathrm{~m}$.

It can be obtained from Figure 16 that when the vector hydrophone and 8103 were deployed together, the signals obtained are correlated, and the delay difference is 0 . When the vector hydrophone and 8103 were deployed separately at a distance of $3.45 \mathrm{~m}$, the delay difference is $0.5 \mathrm{~s}$. The calculated wave velocity is $6.9 \mathrm{~m} / \mathrm{s}$, the velocities of gravity waves and sound waves are $7 \mathrm{~m} / \mathrm{s}$ and $1500 \mathrm{~m} / \mathrm{s}$, respectively. It proves that the signal received by the hydrophone is a gravity wave rather than a sound wave.

Based on the above test results, the hydrophone measures the pressure change through the elastic deformation of the sensitive element. Fluid velocity changes and density changes can cause pressure changes. In very low frequency bands, the pressure signal actually measured by the hydrophone is caused by fluid velocity changes, which is hydrodynamic pressure. It can be assumed that the fluid is an incompressible fluid. Therefore, the AFSD can be measured with a vector hydrophone.

The pressure spectrum at $z=d$ can be obtained through the time domain signal. The relationship between the amplitude of the pressure generated underwater and the amplitude of the surface pressure is $P_{0}=P_{z} \cosh (k h) / \cosh (k(h+d))$, the surface pressure spectrum can be obtained through the relationship. Take $30 \mathrm{~s}$ time domain signal and 
use the Welch periodogram to get the power spectrum. Spectral resolution is $0.0333 \mathrm{~Hz}$. Figure 17 shows environmental noise spectrum and the surface pressure spectrum obtained by the target heave and sway motion at $0.43 \mathrm{~Hz}$. The horizontal axis is the frequency of the hydrodynamic wave, the left vertical axis is the size of the hydrodynamic pressure spectrum, and the right vertical axis is the corresponding pressure spectrum level. The reference pressure is $P *=1 \mu \mathrm{Pa}, 1 \mu \mathrm{Pa}=0 \mathrm{~dB}$, pressure spectrum level is $S(f)=20 \log (P(f) / P *)$. It can be seen from Figure 17 that the environmental noise level is relatively large, which may be due to sensitivity. Since the radius of the sphere is much smaller than the water depth and the sphere is placed in a deeper position, the surface pressure of surface gravity waves are relatively small and the signal-to-noise ratio is relatively low.

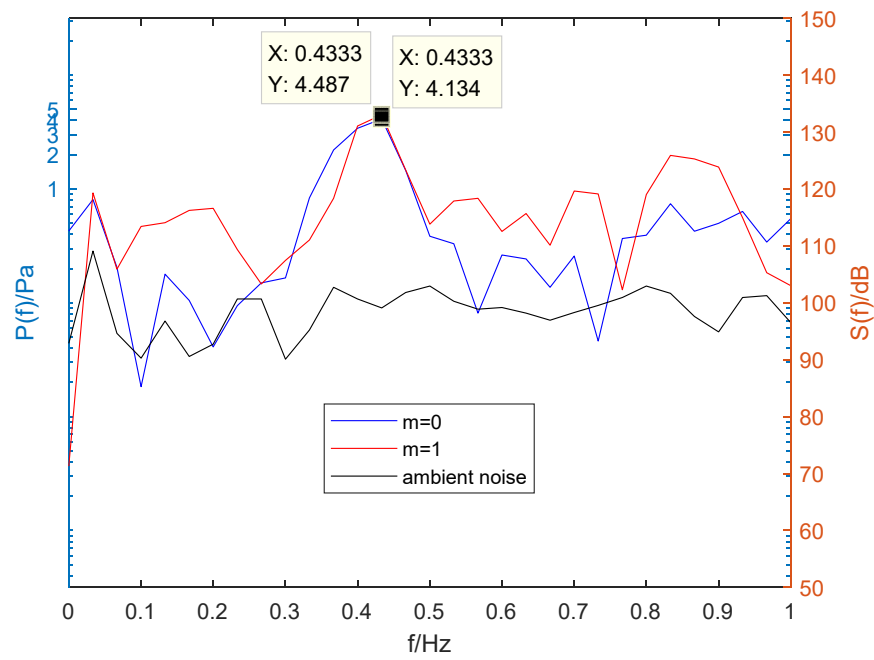

Figure 17. P channel pressure spectrum of vector hydrophone.

The amplitude of the surface pressure can be obtained according to the relationship $p=\rho g \Delta h$. The test result is larger than the numerical result, it may be due to the influence of waves generated by the reflection of the pool wall and the walking mechanism. Figure 18 shows the measured AFSD when the target sways and heaves at $f=0.43,0.5,0.6,0.7,0.9$, and compare it with the normalized numerical simulation results of Figure 9. The result shows that the change trend of the test result is consistent with the simulation result.

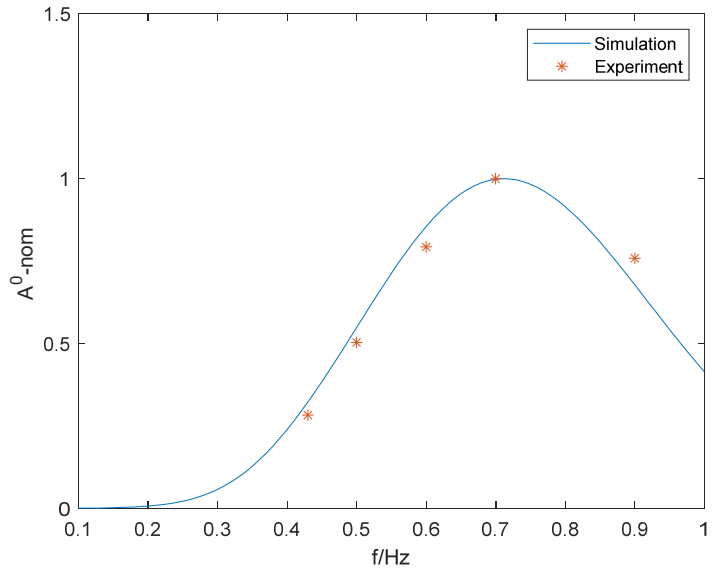

(a)

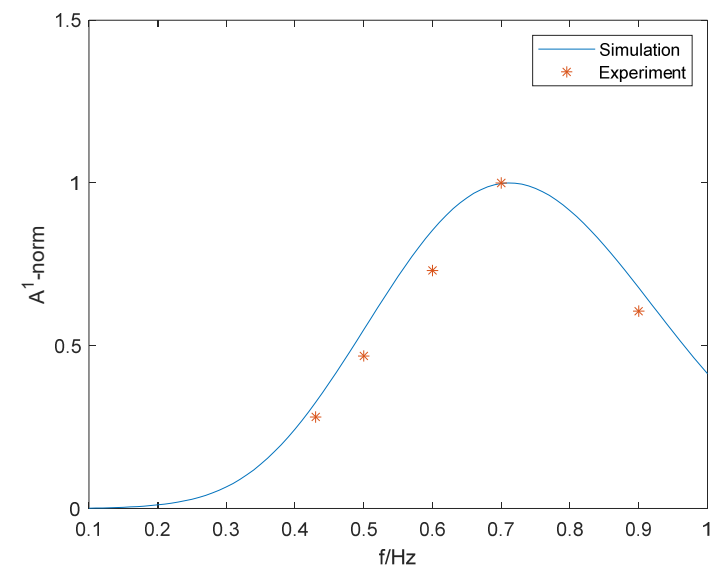

(b)

Figure 18. Normalized AFSD obtained by simulation and experiment. (a) $m=0$, (b) $m=1$. 


\section{Conclusions}

The Karman vortex generated by the underwater moving target will cause periodic oscillation of the structure, which may cause the periodic movement of the surrounding fluid and generate ULF gravity waves. The waves are gravity waves instead of sound waves. In the two-layer fluid, the sphere oscillation at a given frequency will produce gravity waves of surface wave mode and internal wave mode. When the upper fluid depth is 0 , the fluid becomes a single layer, and oscillation of the sphere produces only gravity waves of surface wave mode, namely, surface gravity waves. Surface gravity waves propagate faster than internal waves, which is helpful for underwater target detection. This article only studies the surface gravity wave generated by sphere oscillation.

For the oscillating motion of a sphere in a single-layer and two-layer fluid, it can be decomposed into two modes: heave and sway. Firstly, within the framework of linear potential flow theory, a multipole expansion theory of velocity potential under two oscillating motion modes is established, and an analytical method for calculating the AID of surface gravity waves is proposed. The AID caused by the spherical heave and sway motion first increases and then decreases as the frequency $\mathrm{Ka}$ increases, for a given target depth. Also, large target depths are associated with small AID. The AIID is smaller than AFSD. When the sphere is in the upper fluid, the layering effect of the fluid has negligible impacts on the AFSD caused by the spherical sway and heave motion. On the contrary, when the sphere is in the lower fluid, the layering effect of the fluid has significant impacts on the ASFD. Therefore, it is important to consider the stratification effect of fluid. Meanwhile, the influence of the position of the internal interface on the AID is analyzed. The results show that the position of the internal interface barely affects the ASFD caused by the sphere sway and heave motion. On the contrary, the position of the internal interface has a great influence on the AIID, with the increase of the depth of the internal interface, the AIID decreases. For underwater moving target detection, the AIID is smaller than AFSD, so that the influence of the internal interface position on the AID can be ignored. Finally, the waves generated by the oscillating sphere was tested in a pool. Based on the measurement of the circular wave by the vector hydrophone, the results of the experiment verify that the wave generated by the oscillating sphere is surface gravity wave. Furthermore, comparing the normalized AFSD of experiment and simulation, it is found that the change trend of normalized AFSD is consistent.

Author Contributions: Conceptualization, E.F.; Data curation, H.W. and M.W.; Formal analysis, H.W.; Investigation, H.W. and Z.L.; Methodology, H.W.; Resources, E.F. and L.H.; Software, H.W.; Supervision, E.F. and L.H.; Validation, Z.L. and M.W.; Writing-original draft, H.W.; Writingreview and editing, E.F. and L.H. All authors have read and agreed to the published version of the manuscript.

Funding: This research received no external funding.

Conflicts of Interest: The authors declare no conflict of interest.

\section{References}

1. Liu, B.; Lei, J. Principles of Hydroacoustics; Harbin Engineering University Press: Harbin, China, 2010.

2. Jokel, C.; Yankaskas, K.; Robinette, M.B. Noise of military weapons, ground vehicles, planes and ships. J. Acoust. Soc. Am. 2019, 146, 3832-3838. [CrossRef] [PubMed]

3. Siderius, M.; Gebbie, J. Environmental information content of ocean ambient noise. J. Acoust. Soc. Am. 2019, 146, 1824-1833. [CrossRef] [PubMed]

4. Andrew, R.K.; Howe, B.; Mercer, J.A.; Dzieciuch, M.A. Ocean ambient sound: Comparing the 1960s with the 1990s for a receiver off the California coast. Acoust. Res. Lett. Online 2002, 3, 65-70. [CrossRef]

5. Scholte, J.G. The range of existence of Rayleigh and Stoneley waves. Geophys. Suppl. Mon. Not. R. Astron. Soc. 1947, 5, 120-126. [CrossRef]

6. Rauch, D. Experimental and Theoretical Studies of Seismic Interface Waves in Coastal Waters; Springer: Boston, MA, USA, 1980; pp. 307-327. [CrossRef]

7. Dorman, L.M.; Sauter, A.W.; Bradley, C.; Porras, J. Short-range seismoacoustic propagation on and off the beach. J. Acoust. Soc. Am. 1995, 98, 2971. [CrossRef] 
8. Zhao, H.; Sun, G.; Zhu, H.; Yuan, J. A Method for Estimating the Distance of Near-Ocean-Bottom Sources by Combining VLF Underwater Acoustic Field and Scholte Wave Field. In Proceedings of the 2018 5th International Conference on Coastal and Ocean Engineering (ICCOE 2018), Shanghai, China, 27-29 April 2018; IOP Publishing: Bristol, UK, 2018. [CrossRef]

9. Li, J.; White, P.R.; Roche, B. Seafloor noise ensemble from vessel manoeuvre in the central North Sea. Ocean. Eng. 2020, 196, 106836. [CrossRef]

10. Alekseev, V.N.; Semenov, A.G. Sound scattering by a moving sphere. Akust. Zhurnal. 1992, 38, $789-797$.

11. Semenov, A.G. On optimization possibility for ocean acoustic tomography system. J. Phys. IV 1994, 4, 1087-1090. [CrossRef]

12. Munk, W.H.; Iglesias, H.V.; Folsom, T.R. An instrument for recording ultra low frequency ocean waves. Rev. Sci. Instrum. 1948, 19, 654-658. [CrossRef]

13. Mysak, L.A.; Leblond, P.H. Waves in the Ocean; Elsevier Scientific Publishing Company: Amsterdam, The Netherlands, 1978.

14. Semenov, A.G. Ultra Low Frequency Fields of Moving Bodies; Nova Science Publishers, Inc: New York, NY, USA, 2017.

15. Gortyenko, V.A.; Zhifu, J. Vector-Phase Methods in Acoustics; National Defense Industry Press: Beijing, China, 2014.

16. Xue, F.; Jin, W.; Qiu, S.; Yang, J. Airborne optical polarization imaging for observation of submarine Kelvin wakes on the sea surface: Imaging chain and simulation. ISPRS J. Photogramm. Remote Sens. 2021, 178, 136-154. [CrossRef]

17. Chen, K.; You, Y.; Noblesse, F. Experimental study of quasi-2D dipolar vortex streets generated by a moving momentum source in a stratified fluid. Phys. Fluids 2016, 28, 75105. [CrossRef]

18. Robey, H.F. The generation of internal waves by a towed sphere and its wake in a thermocline. Phys. Fluids 1997, 9, 3353-3367. [CrossRef]

19. Shin, S. Simulation of two-dimensional internal waves generated by a translating and pitching foil. Ocean Eng. 2013, 72, 77-86. [CrossRef]

20. Yeung, R. Radiation and diffraction of waves in a two-layer fluid. In The Twenty-Second Symposium on Naval Hydrodynamics; National Academy of Sciences: Washington, DC, USA, 1999.

21. Ursell, F. On the heaving motion of a circular cylinder on the surface of a fluid. Q. J. Mech. Appl. Math. 1949, 2, 218-231. [CrossRef]

22. Thorne, R.C. Multipole expansions in the theory of surface waves. Math. Proc. Camb. Philos. Soc. 1953, 49, 707-716. [CrossRef]

23. Cadby, J.R.; Linton, C.M. Three-dimensional water-wave scattering in two-layer fluids. J. Fluid Mech. 2000, 423, 155-173. [CrossRef]

24. You, Y.X.; Shi, Q.; Miao, G.P. Hydrodynamic characteristics of a submerged pulsating circular cylinder in a two-layer fluid of finite depth. Ocean Eng. 2007, 19, 1-8.

25. Rahman, M. Simulation of diffraction of ocean waves by a submerged sphere in finite depth. Appl. Ocean Res. 2001, 23, 305-317. [CrossRef] 